\title{
A Proposed Design to Protect Museums of Shrines in Iraq Against Vibrations
}

\author{
${ }^{1}$ Ashraf A.M.R. Hiswa, ${ }^{2}$ Abdulkadhim J. Alabidi and ${ }^{3}$ Mustafa Salman Shubber \\ ${ }^{1}$ Department of Hydraulic Structures and Water Resource, Faculty of Engineering, \\ ${ }^{2}$ Department of Geology, Faculty of Science, \\ ${ }^{3}$ Department of Civil Engineering, Faculty of Engineering, University of Kufa, \\ Kufa, 964 Najaf, Iraq
}

\begin{abstract}
The protection against vibrations for museums of shrines in Iraq has never been discussed before. These museums contain valuable artwork and historical objects that are irreplaceable and some of them belong to more than 1400 years. So, the protection of museums against vibrations is necessary and important to be taken into account in the designing of museums. This study is aimed to review the methods and procedures that were developed to protect the artwork and heritage against vibrations and propose new design for museums. These vibrations could be induced due to different factors which can be divided into internal and external factors. Some of these vibrations can lead the museum's content to be damaged. Different protection strategies are presented in order to eliminate the vibrations effects on museum's contents or decrease them. Some protection procedures are taken into account during the proposed museum's design and others are performed during the construction process.
\end{abstract}

Key words: Museums, vibrations, exhibits, performed, protection, account

\section{INTRODUCTION}

The protection against vibrations for museums of shrines in Iraq has never been discussed before. These museums contain valuable artwork and historical objects such as stone, ceramic, metal, glass, ancient foodstuff and wood etc. Some of artwork and historical objects consist of composite materials such as painted or plastered wood and some of them contain joins like wooden dowels and or ancient glue. Some of these objects belong to more than 1400 years. Mechanical vibrations are considered as an important factor to be taken into account in the protection of collections objects (Valach et al., 2015). These objects are extremely variable in responding to vibrations according to their size, mass distribution, shape and material composition. The level of vibrations is usually variable according to the source of the vibrations, therefore, their effects are variable on objects and some vibrations may induce damage to objects during short or long-term exposure. The damage to collections objects can be in form of increasing stresses and or existing cracks growth (Serotta and Smyth, 2014). Some of these objects are irreplaceable and to protect them from the vibrations effects, all possible sources of vibrations should be studied and then eliminated or reduced to accepted vibrations levels.

\section{MATERIALS AND METHODS}

Vibrations sources: The vibrations affecting museums and their contents are variable according to many factors such as the museum location, museum's structural design and other factors surrounding the museum. Fundamentally, Vibrations affecting museums can be divided into internal and external vibrations according to their sources.

The internal vibrations: The internal vibrations can be induced from two sources. The first one is the visitors walking impact where (Johnson and Hannen, 2015; Thickett, 2002) mentioned in their researches that human bodies can perceive low levels of vibrations that can lead objects to be damaged. The vibrations induced from visitors walking transforms to museum's contents through floors then to either walls holding artwork or counters bearing exhibits. The other source is the shock which is produced from dropping unrestrained objects or walking of an object on shelves under the influence of vibration then falling. Shock is considered as a single, high-speed impulse load that may cause immediate damage.

Corresponding Author: Abdulkadhim J. Alabidi, Department of Geology, Faculty of Science, University of Kufa, Kufa, 964 Najaf, Iraq 


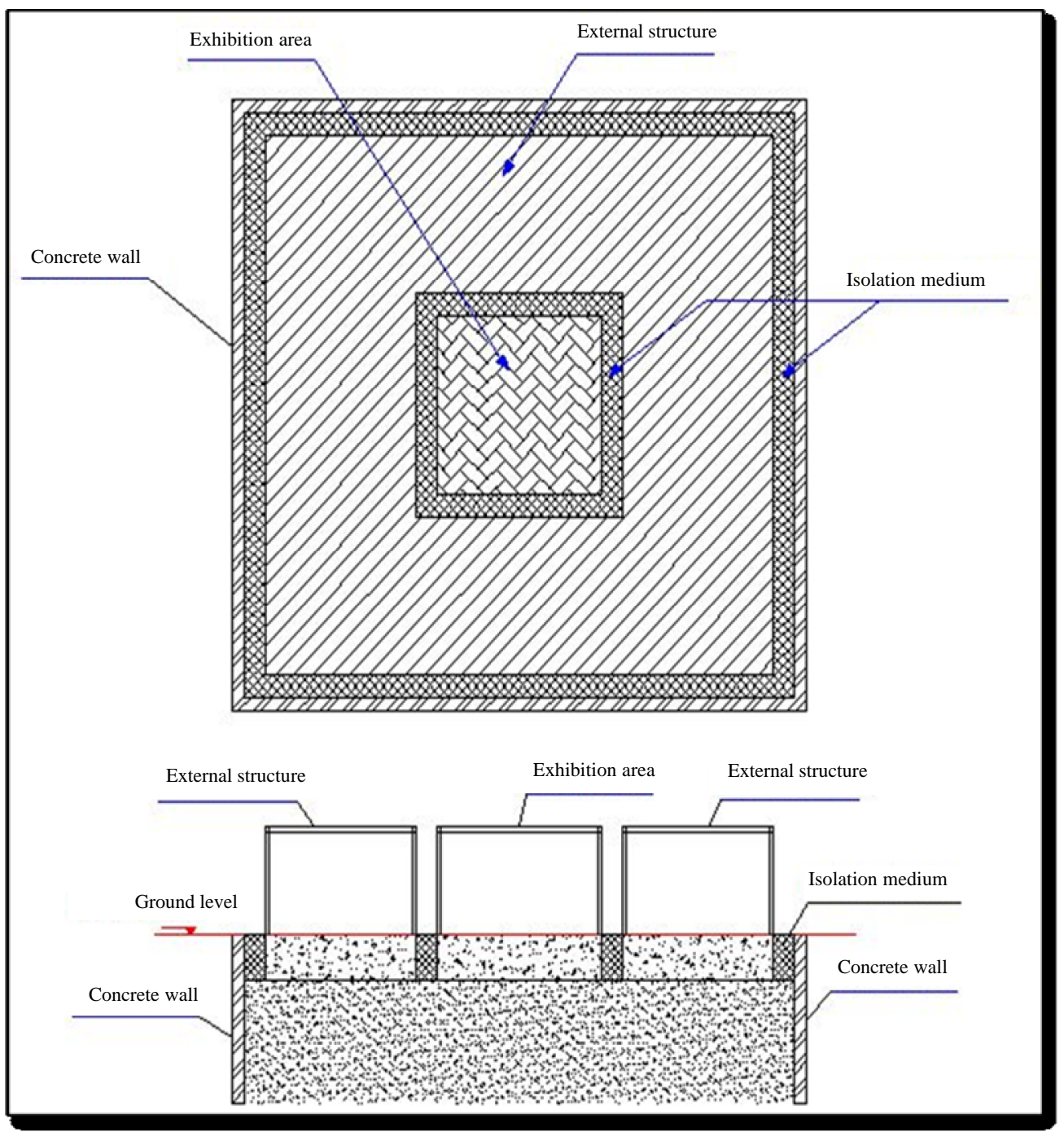

Fig. 1: Proposed isolation of exhibition area

The external vibrations: The external vibrations can be resulted from four main factors as listed.

Traffic vibrations: This type of vibration is due to the movements of different transportations and produces low levels of vibrations in comparison with visitors walking (Valach et al., 2015) (Fig. 1).

Impact of construction and/or demolition vibrations: The vibrations generated by the demolition and or the construction of buildings near or inside a museum are considered high because of the responding of both of the museum's structure and the museum's contents to these vibrations. The studies have been made on this type and recorded high levels of vibrations due to heavy impact tools utilized in the construction process (Serotta and Smyth, 2014). The greatest threat to museum's contents from this type of vibrations is the walking of fragile objects or pre-existing weakness objects on smooth surfaces due to the resonance phenomenon between these objects and the construction vibrations having the same natural frequencies (Johnson et al., 2013).

Earthquakes vibrations: It is so important to take earthquake vibrations into consideration on museums where earthquake can produce high levels of vibrations. Awareness should be spread around the world of the hazard that can be occurs in museums due to earthquakes and mitigation techniques as well as more research are needed to be developed in this scope (Podany, 2015). The 
seismic vibrations can affect the museum structure when the earthquake occurs at the museum region or they can reach the museum structure through Earth toppling when the earthquakes take place far from the museum region (Thickett, 2002). Besides, landslides and mudfloods, resulting from earthquakes, may affect museums in some regions.

Explosions vibrations: This type of vibration source is considered rare and in the same time possible due to the currently unstable situation in Iraq and some other countries. Vibrations due to explosions can be very high and unexpected they could be catastrophic to both of the museum structure and its contents, besides the threat to people's lives.

\section{RESULTS AND DISCUSSION}

Protection of museums from vibrations: The protection strategies for the museums and its contents depend on the vibration source and its intensity. Different strategies can be applied in order to prevent or mitigate the possible vibration damages that could occur to museums and its contents. One of the methods to decrease the potential of damage to the artwork is to reduce the natural amplification of the vibrations that occur in museums. Fundamentally, this involves utilizing a very soft layer, from viscoelastic materials as floors. This strategy can minimize the vibrations induced from the visitors walking and the shock vibrations due to dropping of unrestrained objects or walking of an object on shelves under the influence of vibrations. The museums can also be protected from vibrations generated from external factors by isolating the important parts of museums which contain the artwork (Mondrus et al., 2016). This strategy may be applied by placing pads under the foundations of these parts. Pads can dissipate the energy of the vibrations. Another method of isolation, proposed in this study, can be applied by surrounding the museum building with a concrete wall embedded in the Earth continuing from ground level to lower than museum's foundations. This wall can absorb or mitigate the vibrations transported to the museum through the Earth layers. The exhibition area is separated from the external structure as illustrated in Fig. 1. The external structure contains the corridors, rooms and other museum's facilities. An isolation medium is set between the foundations of all the museums parts and the concrete wall. The role of the isolation medium is to minimize the transformation of vibrations to the exhibition area. This method of isolation can provide a high protection to the important artwork from many external vibrations types such as traffic, impact of construction and or demolition, earthquakes and explosions vibrations. Also, the artwork would be protected from the vibrations induced from visitors walking if the architectural design is perfrmed in way that all the visitors corridors are out of the exhibition area. This can be done by separating the exhibition roof from external museum's structure roof in addition to the foundation separation. The selection of the museum's location plays an important factor in the protection process. The location of the museum should be in place that lies far from the main transportations paths and nearby buildings which could have any future constructions and/or demolitions. Also, museum's location must avoid as possible as hazard locations according to the hazard maps of the area. Furthermore, the security procedure should be in way that reserve the museum and the areas surrounding it from any possible attack that lead to an explosion which affect the museum, its contents and the people attending it.

\section{CONCLUSION}

The protection against vibrations for museums of shrines in Iraq is the aim of this study. These museums contain valuable artwork and historical objects. These objects can be damaged by the mechanical vibrations that could be formed from different factors and in some cases the mechanical vibrations can be a threat for the museum's contents. The vibrations affecting the museums can be formed from both internal and/or external factors. The protection procedures depend on the vibration source. Some protection procedures are performed inside the museum such as utilizing a very soft layer, from viscoelastic materials as floors to minimize the vibrations induced from the visitors walking and the shock vibrations. Some protection procedures are taken into account during the museum's design, proposed this studu in order to preserve the exhibit area by splitting it from the other museum's sections. The selection of the museum's location is considered one of the protection procedures where the location selection can lead to keep the museum far from some vibration sources such as traffic and explosions vibrations. Finally, it can be said that the protection procedures should be taken into account prior to the construction of any museum in order to protect in and its content from damage specially for ancient heritage which is irreplaceable. 


\section{REFERENCES}

Johnson, A.P. and W.R. Hannen, 2015. Vibration limits for historic buildings and art collections. J. Preserv. Technol., 46: 66-74.

Johnson, A.P., W.R. Hannen and F. Zuccari, 2013. Vibration control during museum construction projects. J. Am. Inst. Conserv., 52: 30-47.

Mondrus, V.L., M.D. Dasevskii, V.V. Motorin, D.K. Sizov and S.N. Shtovskii et al., 2016. Vibration protection of cultural heritage (Museum of Private Collections Pushkin Museum Pushkin) from the dynamic effects of the subway: The concept of modeling of process of installation and implementation of vibration protection, measurement. Procedia Eng., 153: 490-495.
Podany, J., 2015. An overview of seismic damage mitigation for museums. Proceedings of the International Symposium on Advances of Protection Devices for Museum Exhibits, April 13-17, 2015, Beijing and Shanghai, China, pp: 1-25.

Serotta, A. and A. Smyth, 2014. Managing construction-induced vibration in the museum environment. Objects Specialty Group Postprints, 21: 263-279.

Thickett, D., 2002. Vibration damage levels for museum objects. J. Conserv., 1: 90-95.

Valach, J., B. Wolf, E. Paulova and S. Urushadze, 2015. Assessment of vibrations in museum's environment. Proceedings of the 21st International Conference on Engineering Mechanics, May 11-14, 2015, Svratka, Czech Republic, pp: 336-337. 